\title{
Opportunistic Sharing between Rotating Radar and Cellular
}

\author{
Rathapon Saruthirathanaworakun, Student Member, IEEE, Jon M. Peha, Fellow, IEEE, and \\ Luis M. Correia, Senior Member, IEEE
}

\begin{abstract}
This paper considers opportunistic primarysecondary spectrum sharing when the primary is a rotating radar. A secondary device is allowed to transmit when its resulting interference will not exceed the radar's tolerable level, in contrast to current approaches that prohibit secondary transmissions if radar signals are detected at any time. We consider the case where an OFDMA based secondary system operates in non-contiguous cells, as might occur with a broadband hotspot service, or a cellular system that uses spectrum shared with radar to supplement its dedicated spectrum. It is shown that even fairly close to a radar, extensive secondary transmissions are possible, although with some interruptions and fluctuations as the radar rotates. For example, at $27 \%$ of the distance at which secondary transmissions will not affect the radar, on average, the achievable secondary data rates in down- and upstream are around $100 \%$ and $63 \%$ of the one that will be achieved in dedicated spectrum, respectively. Moreover, extensive secondary transmissions are still possible even at different values of key system parameters, including cell radius, transmit power, tolerable interference level, and radar rotating period. By evaluating quality of service, it is found that spectrum shared with radar could be used efficiently for applications such as non-interactive video on demand, peer-topeer file sharing, file transfers, and web browsing, but not for applications such as real-time transfers of small files and VoIP.
\end{abstract}

Index Terms - Coexistent, Cooperative, Opportunistic, OFDMA, Primary-secondary spectrum sharing, Radar

\section{INTRODUCTION}

$\mathrm{P}$ RIMARY-SECONDARY spectrum sharing can substantially alleviate spectrum scarcity [1]. Radars could be a good candidate for the primary systems in spectrum sharing because they operate in a large amount of spectrum. For example, in the US over $1.7 \mathrm{GHz}$ of spectrum from $225 \mathrm{MHz}$ to $3.7 \mathrm{GHz}$ "involves radar and/or radionavigation infrastructure," [2] and

Manuscript received January 5, 2012; revised June 3, 2012. This work was supported by the Fundação para a Ciência e a Tecnologia (Portuguese Foundation for Science and Technology) through the Carnegie Mellon Portugal Program under Grant SFRH/ BD/33594/2008.

R. Saruthirathanaworakun is a Ph.D. student in the Carnegie Mellon Portugal program, with Department of Engineering and Public Policy, Carnegie Mellon University, Pittsburgh, PA 15213 USA, and with Instituto Superior Técnico - Technical University of Lisbon, Portugal (phone: $(+351)$ 218-418-199; fax: (+351) 218-418-291; e-mail: rsaruthi@andrew.cmu.edu).

J.M. Peha is with the Dept. of Engineering and Public Policy and the Dept. of Electrical and Computer Engineering, Carnegie Mellon University (e-mail: peha@cmu.edu).

L.M. Correia is with Instituto Superior Técnico/Instituto de Telecomunicações - Technical University of Lisbon (e-mail: luis.correia@1x.it.pt). around $1.1 \mathrm{GHz}$ of this $1.7 \mathrm{GHz}$ is used by fixed land-based radars in non-military applications [3].

This paper considers opportunistic sharing where a secondary device can transmit only when its transmissions will not cause harmful interference, i.e., interference that causes noticeable service disruption to the primary [4].

Currently, there are some bands in which radars are protected from harmful interference by granting them exclusive rights to operate in a given area and frequency band, and other bands (e.g., at $5 \mathrm{GHz}$ ) in which radars share spectrum with unlicensed devices that can transmit only if they are so far from any radar that the radar is undetectable [5]. Both of these are white space approaches to primarysecondary sharing, where secondary wireless systems are allowed to operate in frequency bands and geographic regions that are found to be entirely "unused" by any primary system. In contrast, with the gray space sharing [6] considered in this paper, a secondary device is allowed to transmit near a radar, but only when and with a transmit power that will not cause harmful interference. The maximum transmit power of secondary devices changes over time, based on the behavior of the primary system. We have previously used this approach to prevent a secondary spectrum user from causing harmful interference to a primary user, when the primary was a cellular system [7]. Dynamic power control has long been used to control devices within a single communication system, e.g., a cellular network, but here it is used to allow systems under different administrative control to share spectrum in a primary-secondary arrangement.

We investigate how much the secondary system can transmit with this different sharing concept, by considering sharing with a rotating radar. Examples of these radars include applications in weather operating in $[2.7,3.0] \mathrm{GHz}$, Air Traffic Control (ATC) in $[2.7,2.9] \mathrm{GHz}$, and other surveillance in $[0.42,0.45] \mathrm{GHz}$ and $[2.7,3.5] \mathrm{GHz}$ [3]. With a rotating main beam, the radar antenna gain seen by the fixed secondary device varies, hence, there will be periods of time when the link loss (including antenna gains and path loss) between the device and the radar is high enough so that the device can transmit successfully, without causing harmful interference to the radar. This sharing can be either cooperative (through explicit coordination between the secondary device and the primary system) or coexistent (without coordination) [1].

The case where a secondary system operates in non- 
adjacent cells is considered; two examples are a secondary system providing broadband hotspots, and a cellular system using shared spectrum when a traffic surge temporarily exceeds what can be supported in dedicated spectrum..

Only a few previous studies have addressed spectrum sharing with a rotating radar. Marcus qualitatively discussed the possibility in [8]; Wang et al. [9], and later Rahman and Karlsson [10], analyzed coexistent sharing quantitatively, but only when a device is far enough that its transmissions will not cause harmful interference even in the radar's main beam, as occurs in $5 \mathrm{GHz}$ band. Instead, the proposed sharing makes use of the varying link loss between the radar and a secondary device, hence, a device may transmit at a closer distance to the radar, but with some interruptions and fluctuations in transmissions as the radar rotates.

We quantify the extent of secondary transmissions, and characterize the effect of interruptions and fluctuations on secondary transmissions. Performance is analyzed for six applications from the four classes of services [11]: 1) Voiceover-IP (VoIP, conversational), 2) non-interactive video on demand (streaming), 3) Peer-to-Peer file sharing (P2P, background), 4) automatic meter reading (e.g., electricity meter reading, background), 5) file transfer (interactive, with traffic in both up- and downstreams), and 6) web browsing (interactive, with traffic mostly in downstream).

The sharing scenario is described in Section II. The power level that a secondary device will be allowed to transmit and the resulting SINR (Signal to Interference plus Noise Ratio) are derived in Section III. Parameters used to evaluate performance of the sharing, numerical results, and conclusions are discussed in Sections IV, V, and VI, respectively.

\section{SPeCtrum Sharing SCENARIO}

To quantify the overall extent of secondary transmissions, a canonical case is considered where there is one radar, one secondary Base Station (BS), and one secondary Mobile Terminal (MT), as shown in Fig. 1. The BS is $d_{R d-B S}$ away from the radar, and the MT is $d_{B S-M T}$ from the BS. Both BS and MT are called secondary devices (SDs).

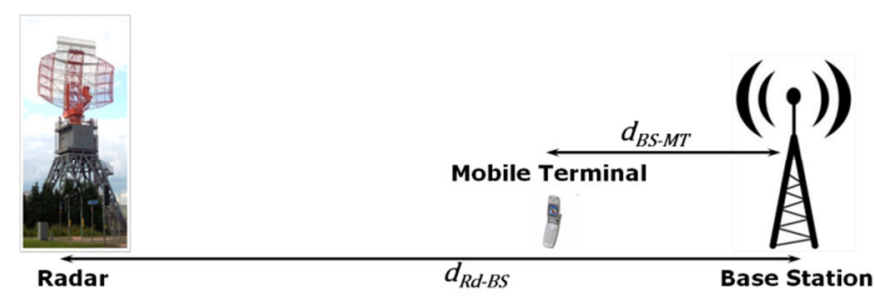

Figure 1 - Sharing Scenario.

The radar uses the same antenna for transmission and reception. The radar's main beam rotates, hence, the radar's radiation pattern seen by an $\mathrm{SD}$, depends on the angle between its main beam and the SD. The rotation can be either mechanical as when a radar antenna rotates, or electronic as when phased array is used. The radar transmits a series of pulses with constant power, and detects echoes from its surroundings [12].
We assume that the SD knows either the instantaneous antenna gain of the radar together with the expected value of path loss between itself and the radar, or the summation of the two, and that the SD can remain synchronized with the main beam rotation. The instantaneous path loss may differ from the expected value due to fading and other factors. The SD can determine this loss in a variety of ways, depending on the sharing approach and type of radar. For example, for a typical ATC radar (rotating horizontally with a constant speed), in a sharing scheme based only on coexistence, the SD may combine any a priori knowledge of the radar with observations over numerous rotations to determine the periodic pattern of the expected link loss, which includes antenna gains of the secondary device and the radar, and the expected path loss. Alternatively, some observations can be replaced by cooperative sharing, which allows the radar to explicitly inform SDs about patterns. The situation is more complicated with a weather radar, which scans in both horizontal and vertical planes at a speed that may change when a storm is approaching [13], or a tracking radar, which may suddenly change the direction of its main beam in response to a target's movements. Cooperative schemes are more useful in such cases. With or without cooperation, the SD should stop transmitting, and resynchronize if the monitored rotation pattern appears to change unexpectedly. The possibility of synchronization errors is beyond the scope of this paper.

A radar insidiously misdetects targets if the interference level is high enough to disrupt its echo receptions [14]. To protect radars from harmful interference, a maximum in-band Interference-to-Noise Ratio (INR) is defined (e.g., by [13]) below which the radar's detection performance is largely unaffected, and INR must be kept below this limit with sufficiently high probability. Many radar systems are used in applications that affect people's lives, so preventing harmful interference in these cases is especially important. For example, recently in the UK there has been discussion on the risk of inadvertent interference to aviation radars operating in the $2.7 \mathrm{GHz}$ band from LTE operating in $2.6 \mathrm{GHz}$ [15].

We assume that the secondary system uses Orthogonal Frequency Division Multiple Access (OFDMA), which is used in wireless communication systems such as LTE. However, the proposed sharing model could be applicable to other secondary systems as well. The proposed sharing model assumes that: 1) Some information of the radar, such as pulse power, rotating period, and tolerable interference level, is known to the secondary system (these parameters rarely change over time); 2) The secondary system: a) uses Time Division Duplex (TDD), b) will use as much available bandwidth as possible, c) can always transmit signaling traffic without causing harmful interference to a radar (this could easily happen, e.g., if signaling is transmitted in a frequency band different from the one shared with the radar).

There are additional assumptions on the secondary applications: 1) signaling traffic is negligible, 2) time used to transfer data within networks (i.e., network delay) is negligible compared to the time used to transfer the data between MT and BS, 3) MTs using video on demand have enough memory 
for buffering, and a constant streaming rate is used.

\section{SeCONDARY MaXimum Allowable TRANSmit Power AND ACHIEVABLE SINR}

An SD determines its maximum allowable transmit power, $P_{S, \text { max }}$, from the radar's tolerable level $I_{t o l}$ (which is known to the SD, assumption 1 in Section II), and the expected link loss between itself and the radar, which varies depending on azimuthal angle between the radar's main beam and the device, $\varphi$. When one secondary transmitter is using as much bandwidth as possible (assumption 2), from $I_{t o l}$, the SD at distance $d_{R d-S}$ from a radar can determine $P_{S, \max }$ as

$P_{S, \max }\left(\varphi, d_{R d-S}\right)=\min \left\{\frac{1}{M_{S}} \frac{I_{\text {tol }}}{\bar{L}_{l, R d-S}\left(\varphi, d_{R d-S}\right)}, P_{S, \text { eqmt_max }}\right\}$,

where $P_{S, \text { eqmitmax }}$ is the SD's transmit power limit, and $\bar{L}_{l, R d-S}\left(\varphi, d_{R d-S}\right)$ is the link loss, between the radar and the transmitter, that the transmitter expects. As discussed in Section II, there are various ways that an SD can determine $\bar{L}_{l, R d-S}$, e.g., when coexistent sharing is employed, $\bar{L}_{l, R d-S}$ could be estimated during a start-up phase by averaging over repeated samples of instantaneous link loss between the radar and the SD. Margin $M_{s}$ provides interference protection for any difference between the instantaneous path loss and the one expected by the SD, including differences from signal fading and from any measurement error during the start-up phase. When the difference from the expected path loss is high, to keep the probability of harmful interference at a particular small level, a higher margin will be needed; this higher margin will result in more conservative extent of secondary transmissions. Even with an adequate margin, gray space sharing inherently introduces some risks that are not presented with white-space sharing, e.g., the power control mechanisms in a secondary system might be hacked and made to interfere with the primary system, or some system bug might cause a secondary transmitter to inaccurately calculate $P_{S, \max }$. To mitigate the effects of interference in such cases, a new approach to regulation and governance is required, which is beyond the scope of this paper, but discussed in [6], [16].

From (1), it is possible to calculate SDs' SINR, $\rho_{S}$, which will in turn determine achievable data rates. This calculation is based on the conservative assumption that the time between radar pulses during which the secondary will not experience interference from the radar (which is typically less than $1 \mathrm{~ms}$ [13]) can be neglected. When a secondary transmitter and receiver are $d_{R d-T X}$ and $d_{R d-R X}$ away from the radar, and the secondary transmitter and receiver are $d_{T X-R X}$ apart, $\rho_{S}$ is

$\rho_{S}\left(\varphi, d_{R d-T X}, d_{R d-R X}, d_{T X-R X}\right)=\frac{L_{l, S}\left(d_{T X-R X}\right) \times P_{S, \max }\left(\varphi, d_{R d-T X}\right)}{n_{S} W_{S}+L_{l, R d-S}\left(\varphi, d_{R d-R X}\right) \times P_{R d}}$

where $L_{l, S}\left(d_{T X-R X}\right)$ is link loss between the secondary transmitter-receiver pair, $n_{S}$ is background noise power spectral density at the secondary receiver, and $W_{S}$ is a secondary bandwidth per user.

\section{PARAmeters For EVAluating THE EXTENT OF SECONDARY TRANSMISSIONS}

The parameters used to evaluate the performance of spectrum sharing with radar are: 1) secondary data rate, $R_{b, S}$, calculated from $\rho_{S} ; 2$ ) fraction of time that the SD can achieve a required data rate $R_{b, r e q}$; and 3) statistics and distribution of time that $R_{b, S}<R_{b, r e q}$, i.e., interrupted time.

From the SINR in (2), the resulting $R_{b, S}\left(\rho_{S}\right)$ is calculated using a set of equations, obtained from regression analyses on 3GPP data [17]. The data are obtained assuming 1) an urban or suburban environments with fairly small cell and low delay spread (i.e., Extended Pedestrian A channel model); 2) a very low speed user (i.e., 5-Hz Doppler frequency); and 3) Multiple Input Multiple Output $2 \times 2$ in both up- and down-streams. The resulting $R_{b, S}\left(\rho_{S}\right)$ will be the maximum rate obtained among QPSK, 16QAM, and 64QAM modulation schemes. From (2), $R_{b, S}$ varies periodically, and is a function of $\varphi, d_{R d-T X}, d_{R d-R X}$, and $d_{T X-R X}$. For a given $R_{b, r e q}$, the interrupted time together with its statistics, and the fraction of time that $R_{b, S} \geq R_{b, r e q}, p_{R_{b, S} \geq R_{b, r e q}}$, in a rotating cycle is:

$$
p_{R_{b, S} \geq R_{b, r e q}}=\frac{\sum_{\forall i}\left[\tau_{R_{b, S} \geq R_{b, r e q, i}}\right]}{T_{R d}},
$$

where $\tau_{R_{b, S} \geq R_{b, r e q, i}}$ is the $i$-th period (in a rotating cycle) that $R_{b, S} \geq R_{b, r e q}$, and $T_{R d}$ is the rotating period.

Different service classes require different quality measurements [11]. For the considered applications, different parameters are used to evaluate performance: 1) VoIP requires a symmetric constant data rate $R_{b, V o I P}$ in both up- and downstreams, and an interrupted time $\tau_{V O I P}$ lower than an acceptable level; 2) Video on demand initially requires an average downstream rate over a rotating cycle $\overline{R_{b, S}}$ larger than the constant streaming rate $R_{b, V i d e o}$; as a buffer is used to maintain streaming continuity, the probability that the buffer will be empty is also evaluated; 3) P2P, file transfer, web browsing, and meter reading, require no minimum data rate. , For P2P and file transfers, achievable data rate is an appropriate measure. For an interactive application like web browsing, webpage download time is our measure.

For VoIP, samples of $\tau_{\text {VoIP }}$ across a rotating cycle, and its distribution, were obtained from instantaneous secondary data rate and $R_{b, \text { VoIP }}$. As VoIP is bi-directional, instantaneous secondary data rate for VoIP is the minimum of the up- and downstream values.

For video on demand, given the amount of data needed for initial buffering, $V_{b f_{\text {_init }}}$, the amount of data buffered at time $t$ after streaming starts (or resumes) $V_{b f}\left(t, t_{s t}\right)$ is

$$
V_{b f}\left(t, t_{s t}\right)=V_{b f_{-} \text {init }}+\int_{t^{\prime}=t_{s t}}^{t} R_{b, S, D}\left(t^{\prime}\right) d t^{\prime}-R_{b, \text { Video }},
$$

where $R_{b, S, D}\left(t^{\prime}\right)$ is the instantaneous downstream secondary 
data rate, and $t_{s t}$ is the streaming starting time.

From (4), for a given start time $t_{s t, i}$, one can determine if and when the buffer would be empty. Based on multiple values of $t_{s t, i}$ across a rotating cycle, the probability that the buffer will be empty, i.e., probability of interruptions, $p_{\text {intrp }}$, during streaming can be obtained from:

$$
p_{\text {intrp }}=\frac{\sum_{i=1}^{N_{t_{s t}}}\left[v_{i}\right]}{N_{t_{s t}}}
$$

where $v_{i}$ is a binary variable that equals 1 if $V_{b f}=0$, and 0 otherwise. $N_{t_{s t}}$ is the total number of $t_{s t, i}$ considered. Note that video on demand operates when $\overline{R_{b, S}}>R_{b \text {,Video }}$, hence, if $V_{b f}\left(t, t_{s r t, i}\right) \neq 0$ in a rotating cycle, it will never go to zero during streaming.

For P2P, file transfer, web browsing, and meter reading, a wide range of file sizes is transferred. Because secondary transmissions can be interrupted, files of different sizes experience different perceived data rates $R_{b, S, p}\left(t_{s t}\right)$, which depend on when a file is transferred $t_{s t}$.

$$
R_{b, S, p}\left(t_{s t}\right)=\frac{V_{f}}{T_{f}\left(t_{s t}\right)}
$$

where $V_{f}$ is size of a file, and $T_{f}\left(t_{s t}\right)$ is the time that an SD uses to transfer the file, i.e. file transfer time. Distribution of $R_{b, S, p}$ can be obtained by choosing different $t_{s t}$ 's.

\section{NUMERICAL RESULTS}

Parameters used to obtain numerical results are summarized in Section A. The performance of secondary transmissions averaged across a cell is evaluated in Section B; this average performance is measured in terms of both mean data rate and percentage of time that a secondary device can transmit. Performance for video on demand and VoIP are evaluated in Sections C and D, respectively. The characteristics and performance of secondary transmissions for file upload- and downloading services are shown in Section E. We investigate sensitivity of these results on important system parameters including (secondary) cell radius, radar transmit power, radar tolerable interference level, and radar rotating period in Section F.

\section{A. Scenario}

For the radar we assume: a) operating band at $2.8 \mathrm{GHz}$ with $3 \mathrm{MHz}$ bandwidth (ATC radars operate in this band) [13]; b) 30-m high antenna, uniformly-distributed aperture type, with elevation and azimuthal $3-\mathrm{dB}$ beam widths of $4.7^{\circ}$ and $1.4^{\circ}$, respectively [13], and front-to-back ratio of $38 \mathrm{~dB}$ [12]; c) maximum antenna gain of $33.5 \mathrm{dBi}$ [13], with $5 \mathrm{~dB}$ reduction in the horizontal direction because the antenna is up-tilted to reduce ground reflected signals [18]; d) rotating period $T_{R d}=4.7 \mathrm{~s}$; e) transmit power $P_{R d}=0.45 \mathrm{MW}[13]$; f) INR = $10 \mathrm{~dB}$ [14], with $-106 \mathrm{dBm}$ background noise [13].

For the secondary system, we take: a) symmetric TDD; b) MT and BS with omnidirectional antenna with $0 \mathrm{dBi}$ and sectorized one with $18 \mathrm{dBi}$ [19], respectively; c) cell radius (in a urban or suburban areas) $R=800 \mathrm{~m}$; d) power limit $P_{S, \text { eqme_max }}$ of the $\mathrm{MT}$ and the $\mathrm{BS}$ of 23 and $46 \mathrm{dBm}$, respectively [19]; e) background noise spectral density at the receiver, $n_{S}=-174 \mathrm{dBm} / \mathrm{Hz}$, with $5 \mathrm{~dB}$ noise figure [19].

Concerning the applications: the required rate for VoIP $R_{b, \text { VoIP }}$ is $15 \mathrm{kbps}$ [11]; video on demand requires streaming rate $R_{b \text {,Video }}$ of $1.6 \mathrm{Mbps}$ [20], and will initially buffer content enough to play for $2 \mathrm{~s}$ [21].

The ITU-R P.1546 path loss model [22] between the radar and the secondary system is adopted. The path loss model is valid in the frequency range $[0.03,3] \mathrm{GHz}$, and distances in $[1,1000] \mathrm{km}$. Conservatively, flat terrain is assumed; even though the assumption would result in longer distances that the radar and the secondary can affect each other, it will increase interference between the radar and the secondary system, and reduce the extent of secondary transmissions. For the path loss between a BS and MT, the COST 231 WalfischIkegami model [23] is adopted as follows: a) 1.7-m high MT antenna; b) building height of $15 \mathrm{~m}$; c) 30-m high BS antenna, implying that the cell radius can be up to $1.5 \mathrm{~km}$ [19]. In the US, a nationwide mean height of a commercial cellular tower is around $60 \mathrm{~m}$ (the tower portfolio is from a major US tower company, American Towers [24, Table 1]), hence, $30 \mathrm{~m}$ represents a reasonable compromise between an on-tower antenna and a rooftop one; d) other required parameters as suggested in [23].

Rayleigh fading is assumed in the link between the radar and the MT; as the difference between the height of radar and that of the MT is typically large, Line of Sight (LoS) between both rarely exists. The corresponding margin $M_{s}$, in (1), of $8.4 \mathrm{~dB}$ is used. The margin results in a probability of harmful interference less than $0.1 \%$ [25], which is less than radar's required misdetection probability of around 1\% [26]. For the link between the radar and the BS, Ricean fading (with a $\mathrm{K}$ factor of $10 \mathrm{~dB}$, the corresponding margin resulting in a probability of harmful interference less than $0.1 \%$ is around 5 $\mathrm{dB}$ [25]) is assumed when their separation is less than an LoS distance, and Rayleigh one is assumed otherwise. (With the considered heights of radar and $\mathrm{BS}$, the LoS distance is around $20.8 \mathrm{~km}$, [27, Chapter 2].) The difference between instantaneous link loss, and the value expected by a secondary transmitter $\bar{L}_{l, R d-S}$, as shown in (1), that is due to measurement error, is assumed to be negligible compared to the difference due to fading.

To evaluate a typical extent of secondary transmissions achievable in a cell, data rate and percentage of time that a secondary device can transmit, averaged across a cell, need to be considered. Hence, the case when the location of the secondary user is uniformly located across the cell is considered. In contrast, to evaluate the performance of sharing for a given application, the location of a secondary user needs to be specified, and the worst case is considered when the user is fixed at the cell edge in the worst direction, which is toward the radar.

For sensitivity analysis, the ranges of parameters considered 
are: a) cell radius $R$ in $[0.2,1.5] \mathrm{km}[19]$; b) radar transmit power $P_{R d}$ in $[0.025,1.4] \mathrm{MW}[13]$; c) radar INR in [-13, 7] $\mathrm{dB}$, adapted from [14]; d) radar rotating period $T_{R d}$ in $[4,6] \mathrm{s}[13]$.

\section{B. Overall Performance of Secondary Transmissions}

Fig. 2 shows the mean secondary data rate over a rotating cycle, and the fraction of time that an SD can transmit, as a function of the distance between the BS and the radar. The data rate and the fraction of transmission time are averaged across the cell.

Fig. 2 shows that spectrum shared with a radar can support high average data rates, even when an SD is close to the radar. Consider the conventional non-opportunistic approach. The distance between the BS and the radar that allows the SD to transmit all the time at the data rate achieved in dedicated spectrum, i.e., system rate limit, is around $400 \mathrm{~km}$ (the required separation is around $100 \mathrm{~km}$ for downstream, and $400 \mathrm{~km}$ for upstream). Alternatively, if only the radar is to be protected [9], with a $8.4 \mathrm{~dB}$ fade margin, the minimum separation is $286 \mathrm{~km}$. In contrast, by taking advantage of main beam rotation, significant downstream transmissions are possible at a fraction of this $400 \mathrm{~km}$ distance. At $50 \mathrm{~km}$ from the radar (i.e., just $12.5 \%$ of $400 \mathrm{~km}$ ), in the downstream the SD can transmit almost all the time with an average rate near the system limit of $10.8 \mathrm{Mbps}$. In the upstream, at $19 \%$ of $400 \mathrm{~km}$, the SD can transmit $90 \%$ of the time, with an average rate around $62.5 \%$ of the $8.0 \mathrm{Mbps}$ limit.

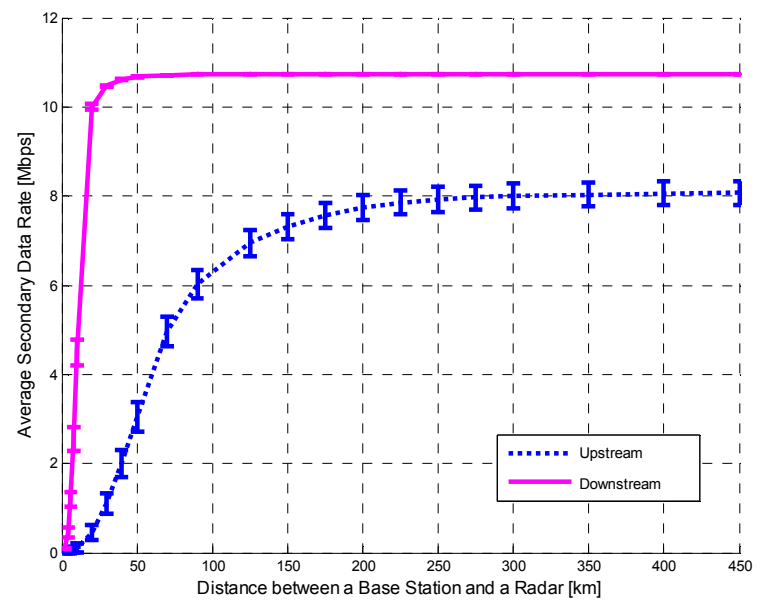

(a) Average Secondary Data Rate with $95 \%$ Confidence Interval

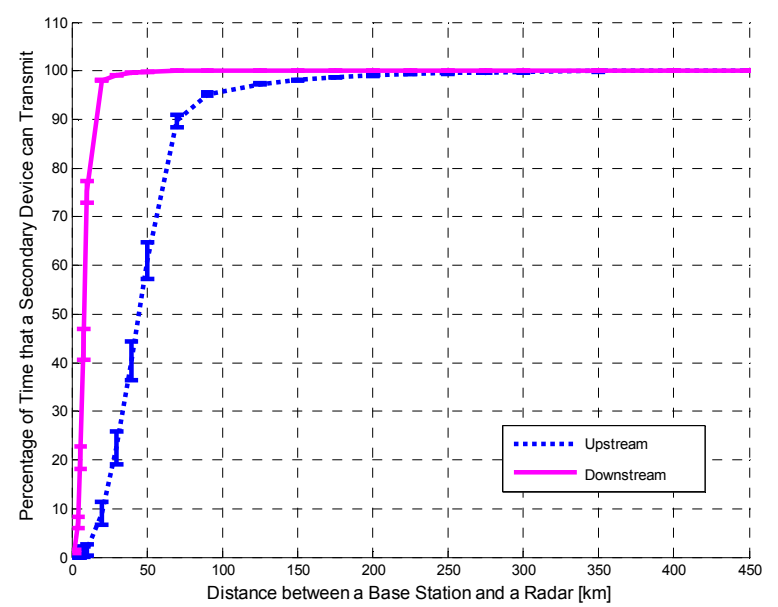

(b) Percentage of Time that a Secondary Device can Transmit with 95\% Confidence Interval

Figure 2 - Extent of Secondary Transmissions vs. Distance between a Base Station and a Radar.

Fig. 2 also shows that the secondary system uses spectrum more efficiently in the downstream than in the upstream, in terms of data rate per $\mathrm{MHz}$ of spectrum, and fraction of time that the system can transmit. If the goal is to maximize spectral efficiency, spectrum sharing with radar might be more suitable for applications that have more traffic in the downstream, which is a typical characteristic of many current applications.

\section{Performance of Video on Demand}

One important measure of performance for video on demand is average downstream data rate. In the previous section, we showed that even at small distance from the radar, the achievable average downstream rate is very close to the rate one might get in dedicated spectrum.

Another performance measurement is the probability that streaming will be interrupted. It is found that this probability is sufficiently low, being unlikely to be a problem. Even when the average downstream rate $\overline{R_{b, S}}$ is only $4 \%$ higher than the streaming rate, and the BS is $9.6 \mathrm{~km}$ from the radar, with the initial buffering of $2 \mathrm{~s}$ the possibility of interruption is less than 0.001 . (The hypothesis testing on this probability used 10,000 samples; the resulting p-value is 0.0008 ; the result is obtained when the MT is at the cell edge closest to the radar.) From the assumption on the initial buffering, the amount of content the application needs to initially buffer for $1.6 \mathrm{Mbps}$ streaming rate is $400 \mathrm{kB}$. With only $3 \mathrm{MHz}$ of spectrum, the transfer time for the initial-buffer content is at most $3 \mathrm{~s}$ even at only $9.6 \mathrm{~km}$ from the radar. Graphs are omitted for brevity.

Due to the high achievable average downstream data rate, and the small chance of being interrupted (even with only a few seconds of initial buffering, and when the secondary is very close to the radar), video on demand is a very promising application for spectrum that is shared with radar.

\section{Performance of Voice over IP}

One important requirement for VoIP is a symmetric data rate in both up- and downstreams. Hence, the asymmetric 
data rate, as shown in Fig. 2(a), will limit performance of VoIP. Moreover, VoIP performance depends on instantaneous data rate, and the application cannot tolerate long interruptions. In shared spectrum, interruptions sometimes cause the instantaneous secondary data rate to be much lower than the average one, and could be a problem for VoIP.

With a required instantaneous rate of $15 \mathrm{kbps}$, Fig. 3 shows the probability that the resulting interrupted time $\tau_{V o I P}$ would be less than an acceptable level $\tau_{\text {VoIP } a}$ (when the user is at the cell edge), as a function of distance of secondary transmissions from a radar; $\tau_{V O P_{-} a}$ is taken as 80 and $150 \mathrm{~ms}$ [11]. Fig. 3 shows that $\tau_{\text {VoIP_a } a}$ is always satisfied beyond $70 \mathrm{~km}$ from the radar. Note from Fig. 2(a) that, at this distance, the average upstream rate is around $5 \mathrm{Mbps}$; however, the application can only obtain at least $15 \mathrm{kbps}$ instantaneous data rate with the acceptable interrupted time. Compared to VoIP operating in dedicated spectrum, VoIP is relatively inefficient in spectrum shared with radar, and hence is not attractive for such sharing.

\section{E. Performance of File Up- and Downloading}

As occurs with VoIP, the fluctuations in data rate can also affect performance of file up- and downloading services. Files with different sizes would experience different ranges of perceived data rate, defined in Section IV. The fluctuation and its implications on applications in this service class are investigated, in Sections E-1 and E-2, respectively.

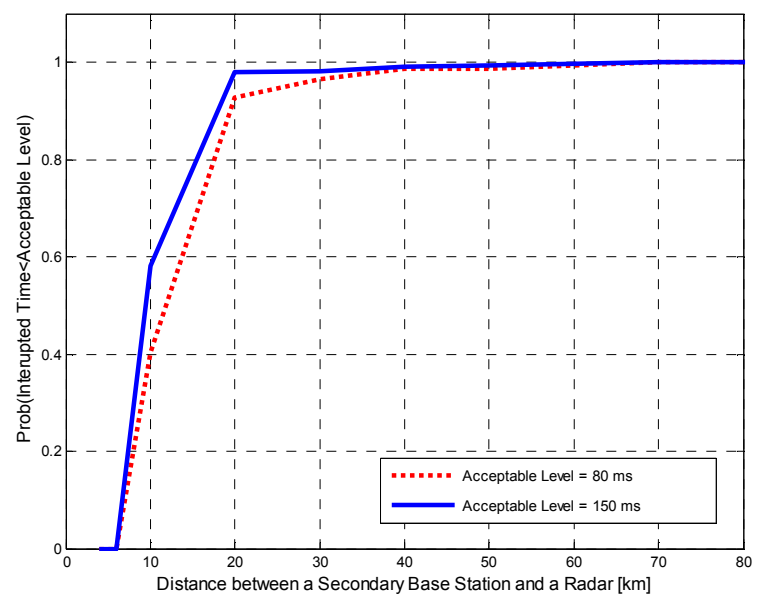

Figure 3 - Possibility that a VoIP Interrupted Time is less an Acceptable Level vs. Distance between a Base Station and a Radar.

\section{E-1 Fluctuations in Secondary Perceived Data Rate}

Fig. 4 shows maximum, mean and minimum perceived downstream data rate of a user at the cell edge as a function of file size. The results from the upstream show a similar trend, and thus are omitted.

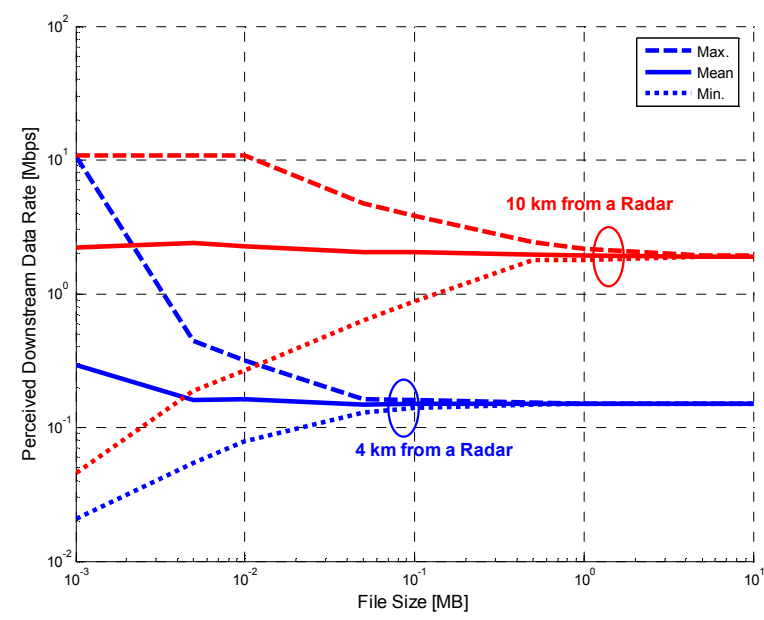

Figure 4 - Perceived Secondary Downstream Data Rate vs. Size of a File.

Fig. 4 shows that a small file would experience a wider range of perceived data rate than a large one, because a small file is more likely to be transferred in less than a rotating period. Due to sporadic interruptions in transmissions, file transfer time, and hence, perceived data rate, would highly depend on when the transfer starts. In contrast, the fluctuations are unnoticeable and appear the same as if this were dedicated spectrum when the file size is above a certain threshold that makes file transfer time much longer than the rotating period; this threshold is much smaller for SDs that are closer to the radar. From Fig. 4, at $4 \mathrm{~km}$ from the radar, the fluctuation in perceived data rate tends to be insignificant for any file larger than $100 \mathrm{kB}$, while at $10 \mathrm{~km}$, the fluctuation starts to be insignificant for a file larger than $1 \mathrm{MB}$.

Thus, fluctuations in perceived data rate depend on file size, and distance between an SD and the radar. As long as radar transmissions still affect secondary transmissions, these fluctuations will be most noticeable when an SD far from the radar transfers a small file. The fluctuations could be a problem for some applications, but not all.

\section{E-2 Implications of the Fluctuations for Various Applications}

We start with P2P and transfers of a large file (e.g., a song which is typically much larger than $1 \mathrm{MB}$ ), for which the perceived data rate is the typical measure of performance. P2P is often used for transfers of large files, and transfers that do not have strict delay requirements. Because file size is large, interruptions in secondary transmissions would have little impact on perceived secondary data rate, as shown in Fig. 4. Moreover, Fig. 2(a) shows that the SD could achieve a high average data rate even close to the radar, hence, $\mathrm{P} 2 \mathrm{P}$ and transfers of large files could also be promising for spectrum sharing, although it might not be quite as promising as video on demand, of which traffic is mostly in the downstream.

Guaranteeing high data rates for small files is more challenging. Fig. 5 shows the 1st-percentile and mean perceived downstream rate (of a user at the cell edge) as a function of distance of a BS from the radar. Fig. 5 shows that transferring files with different sizes would experience approximately the same mean perceived rate at any distance 
from the radar. However, for small files (i.e., smaller than $1 \mathrm{MB}$ ), the 1st-percentile perceived rate can be much lower than the mean, hence, for transfers of small files, this could be a problem, if users would not tolerate fluctuations in perceived data rate. Thus, such applications would not be suitable for sharing spectrum with radar. Upstream results are similar, but are omitted.

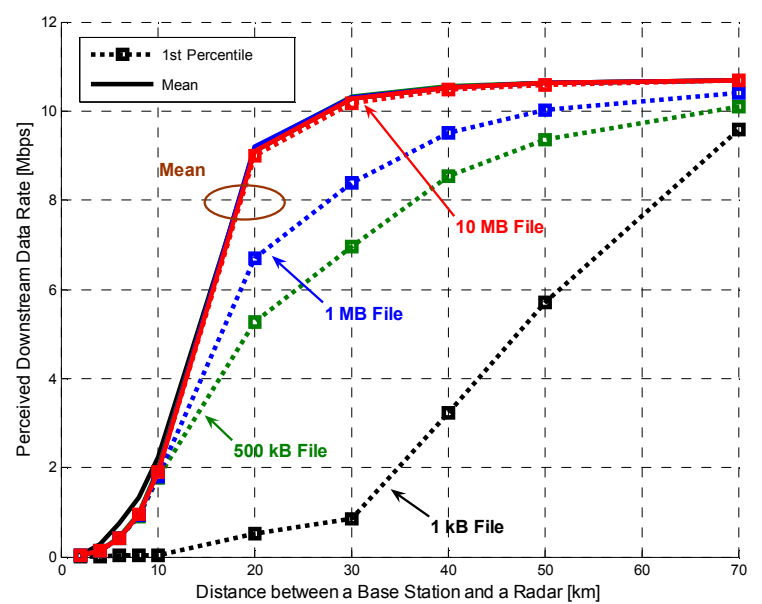

Figure 5 - First Percentile, and Mean Perceived Downstream Data Rate vs. Distance between a Base Station and a Radar.

There are also applications for which file transfer time, rather than perceived data rate, is the important performance measure. Although transferring small files, the applications would still work well in spectrum shared with radar. One example is web browsing, since users probably expect a web page to be retrieved just as quickly, even if it contains many more bytes. Time for downloading a web page is suggested to be less than 2 to $4 \mathrm{~s}$ (with a preferred target of $0.5 \mathrm{~s}$ ) [11].

Fig. 5 shows that just beyond $10 \%$ of the $286 \mathrm{~km}$ distance at which secondary transmissions will not affect the radar, a user downloading a $1 \mathrm{MB}$ web page would experience the 1stpercentile perceived rate of around $8 \mathrm{Mbps}$. The 90thpercentile webpage size in 2010 was $660 \mathrm{kB}$ [28], hence, even with $3 \mathrm{MHz}$ of spectrum, $99 \%$ of the time, more than $90 \%$ of transfers would experience file transfer time less than $1 \mathrm{~s}$. For the web pages larger than $1 \mathrm{MB}$, the file transfer time would be less than $1 \mathrm{~s}$; however, this transfer time is not very different from the one when transmissions occur in dedicated spectrum. For example, with the $10.8 \mathrm{Mbps}$ downstream rate limit, the transfer time for a web page larger than $1 \mathrm{MB}$ would be larger than $740 \mathrm{~ms}$. Thus, quality of service is good for web browsing even close to the radar.

There are also applications that transfer small files, but can tolerate interruptions during transmissions, e.g., automatic meter reading. Generally, the system consists of many meters installed at users' premises; each of these devices intermittently transfers a small amount of information, in the order of tens of $\mathrm{kB}$, to an aggregation point. Because significant delays are tolerable, an average amount of data that can be transferred in a given period is the important performance measure. As Fig. 2(a) shows that the secondary system can achieve high average data rate, spectrum shared with radar would also work well with this application.

\section{F. Sensitivity Analysis}

In this section, we evaluate the sensitivity of the average extent of secondary transmissions, and fluctuations in perceived secondary data rate on four important parameters: secondary cell radius, $R$; radar transmit power, $P_{R d}$; radar tolerable interference level, i.e., maximum INR); radar rotating period, $T_{R d}$.

\section{F-1 Sensitivity of Average Extent of Secondary Transmissions}

Unlike the other parameters considered, changing radar rotating period will not change the amount of data an SD can transfer in a given period of time, and thus, it will have no impact on the average extent of secondary transmissions. Hence, we will omit these graphical results. The average extent of secondary transmission is calculated across a cell.

Fig. 6 shows the average secondary data rate, and the percentage of time that an SD can transmit for both up- and downstream, as a function of the cell radius. Fig. 6 shows that, as expected, the average data rate and percentage of time that a secondary device can transmit at a given distance from the radar decreases with increasing cell radius. It is still the case that extensive communications are possible for a cell that is relatively close to the radar, but for larger cells, the cell must be somewhat farther from the radar.

Fig. 6(a) shows that in the upstream, at $70 \mathrm{~km}$ from the radar, the average data rate of a cell with $0.8 \mathrm{~km}$ radius is around $60 \%$ of the system rate limit, as defined in Section VB; $70 \mathrm{~km}$ is around $24 \%$ of the $286 \mathrm{~km}$ distance. For a larger cell, with $1.5 \mathrm{~km}$ radius, the same level of upstream data rate can be achieved at around $100 \mathrm{~km}$ from the radar $35 \%$ of $286 \mathrm{~km}$ ). Moreover, in the downstream, at only $14 \%$ of the $286 \mathrm{~km}$ distance, a cell with $1.5 \mathrm{~km}$ radius can achieve almost $100 \%$ of the system rate limit. Hence, the secondary system can still achieve high data rates close to the radar, even with a fairly large cell.

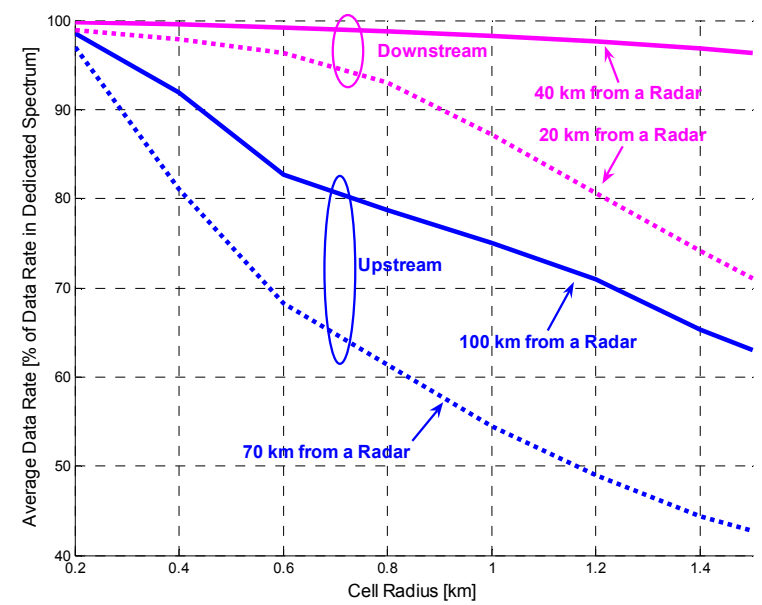

(a) Average Secondary Data Rate 


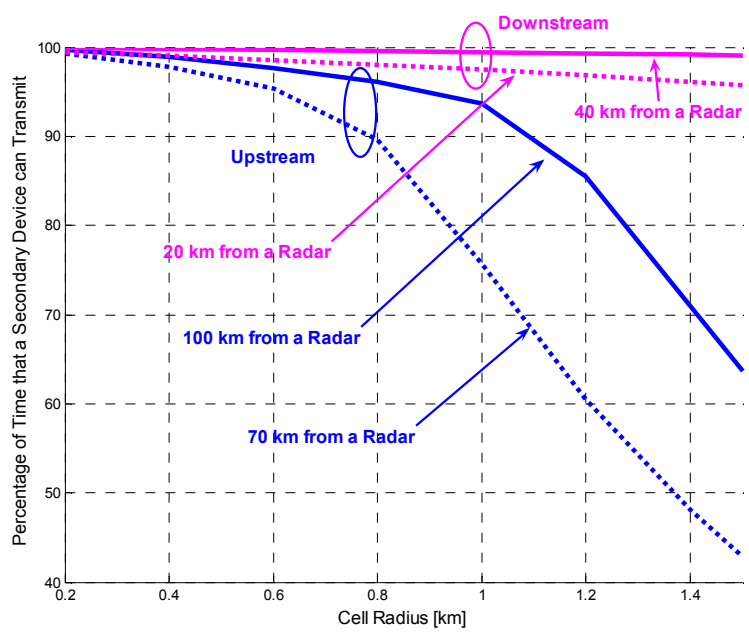

(b) Percentage of Time that a Secondary Device can Transmit Figure 6 - Sensitivity of Extent of Transmissions on Cell Radius.

Fig. 7 shows the average data rate as a function of radar transmit power. Changes in radar transmit power have a similar effect on both data rate and the percentage of time that an SD can transmit, similar to what we observed with changes in cell radius in Fig. 6. Hence, results showing the percentage of time that an SD can transmit are omitted for brevity.

Fig. 7 shows that the data rate decreases with increasing transmit power of the radar. However, high average data rates close to a radar can still be attained, even when the radar transmit power is high, if the cell is a bit farther from the radar. For example, in the upstream when the radar transmit power increases from 0.5 to $1.4 \mathrm{MW}$, the distance from a cell to the radar needs to be increased from around 70 to $90 \mathrm{~km}$, so that the achievable data rate is still around $60 \%$ of the system rate limit $(90 \mathrm{~km}$ is still only $31 \%$ of $286 \mathrm{~km})$. In the downstream, the increase in radar transmit power only slightly decreases the average data rate, hence, the secondary system can achieve high data rates at fairly short distance to a radar transmitting with high power.

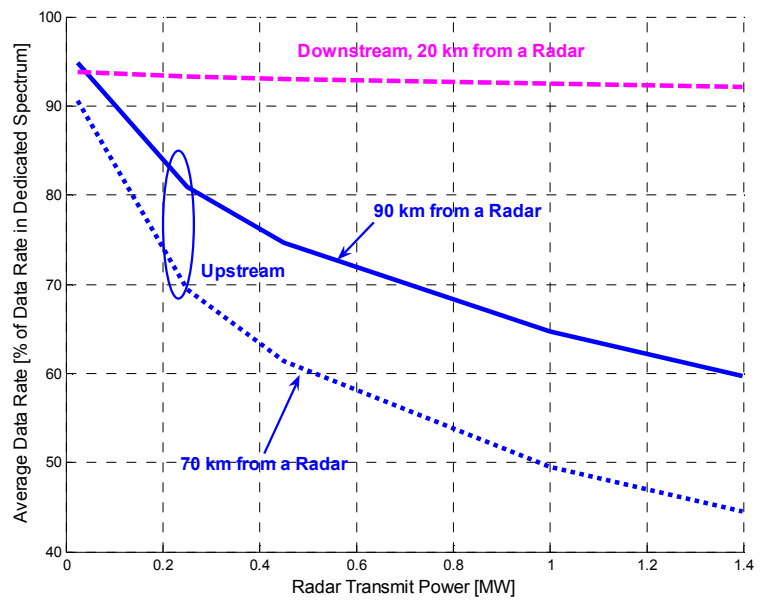

Figure 7 - Sensitivity of Average Data Rate on Radar Transmit Power.

Fig. 8 shows the averaged data rate as a function of the radar's tolerable INR. As expected, the data rate slightly decreases with a more stringent requirement on radar tolerable interference level, i.e., smaller tolerable INR. For example, in the downstream at $20 \mathrm{~km}$ from the radar, reducing INR from 10 to $-13 \mathrm{~dB}$ would decrease the average data rate from $93 \%$ to $88 \%$ of the rate limit. Note from Fig. 8 that we see no impact of INR on the average upstream data rate, because an $\mathrm{SD}$ can transmit with power higher than its equipment limit without causing harmful interference to a radar. Hence, an SD will still transmit at its equipment power limit even when the radar tolerable interference level changes; this is more likely to happen when there are only a few MTs in a cell, as assumed in this scenario. However, when there are many users, we expect that reducing radar's INR would decrease the data rate in both up- and downstreams.

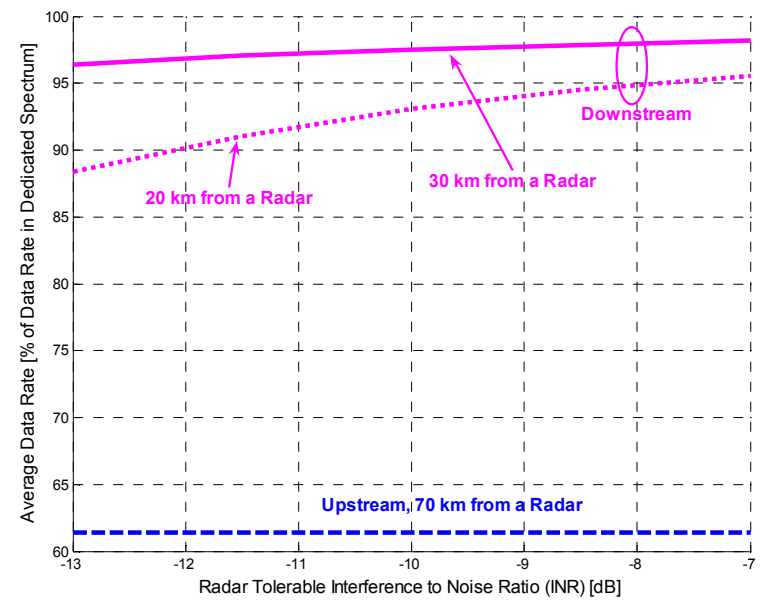

Figure 8 - Sensitivity of Average Data Rate on Radar Tolerable Interference Level.

Similarly to Fig. 6 and Fig. 7, the secondary system can still achieve high data rates under more stringent INRs when a cell is a bit farther from the radar, e.g., by moving a cell from 20 to only $30 \mathrm{~km}$ from the radar, the downstream data rate, for INR $=-13 \mathrm{~dB}$, is $96 \%$ of the rate limit. Thus, the secondary system can also achieve high data rates close to the radar even under radar's stringent tolerable interference requirements.

In addition, for a typical range of cell radius, transmit power and tolerable interference level of the radar, comparing Fig. 6, Fig. 7 and Fig. 8 shows that cell radius would relatively have higher impact on the extent of secondary transmissions compared to the other parameters. For example, at $20 \mathrm{~km}$ from the radar, in the downstream, the average data rate increases from $70 \%$ to $99 \%$ of the rate limit, when cell radius decreases from 1.5 to $0.2 \mathrm{~km}$. At the same distance from the radar, the average downstream data rate increases from $88 \%$ to $96 \%$ of the rate limit, when INR increases from -13 to $-7 \mathrm{~dB}$, while the average downstream data rate slightly increases from $93 \%$ to $94 \%$ of the rate limit, when the radar transmit power decreases from 1.4 to $0.025 \mathrm{MW}$. Hence, at least for an ATC radar, if one adjusts design parameters within a range that might be considered reasonable, the extent of secondary transmissions achievable seems to be affected more by the radius of the cell rather than by the transmit power and tolerable interference of the radar that the secondary system 
will share spectrum with.

\section{F-2 Sensitivity of Fluctuations in Perceived Secondary Data Rate}

Fig. 9 shows fluctuations in perceived data rate as a function of file size: in the upstream at $70 \mathrm{~km}$ from the radar, and in the downstream at $20 \mathrm{~km}$. The fluctuations in perceived data rate are evaluated for a user at the edge of a cell in the direction toward the radar. It is seen, from Section V-E, that when radar transmissions still affect transmissions of the secondary system, the farther away from the radar, the higher fluctuations in perceived data a secondary user would experience. At $70 \mathrm{~km}$ from the radar for upstream and at $20 \mathrm{~km}$ for downstream, secondary transmissions are still highly affected by the radar; Fig. 2 shows that the increasing rate (i.e., slope) of the extent of secondary transmissions with distance from the cell to the radar starts to decrease after around 70 and $20 \mathrm{~km}$ for up- and downstreams, respectively.

Fig. 9(a) shows that, as expected, a user in a smaller cell would experience higher fluctuations in perceived data rate than that in a larger cell. However, the increase in fluctuations in perceived data rate with decreasing cell radius will not be a problem, as a user uploading large files (i.e., larger than $1 \mathrm{MB}$ ) would still experience insignificant fluctuations even when the cell radius is as small as $200 \mathrm{~m}$. For the downstream, Fig. 9(b) shows that at only $20 \mathrm{~km}$ from the radar, a user downloading large files would also experience insignificant fluctuations in perceived data rate even when the cell radius is $200 \mathrm{~m}$. Hence, a user transferring a large file would still experience insignificant fluctuations in perceived data rate even in a small cell.

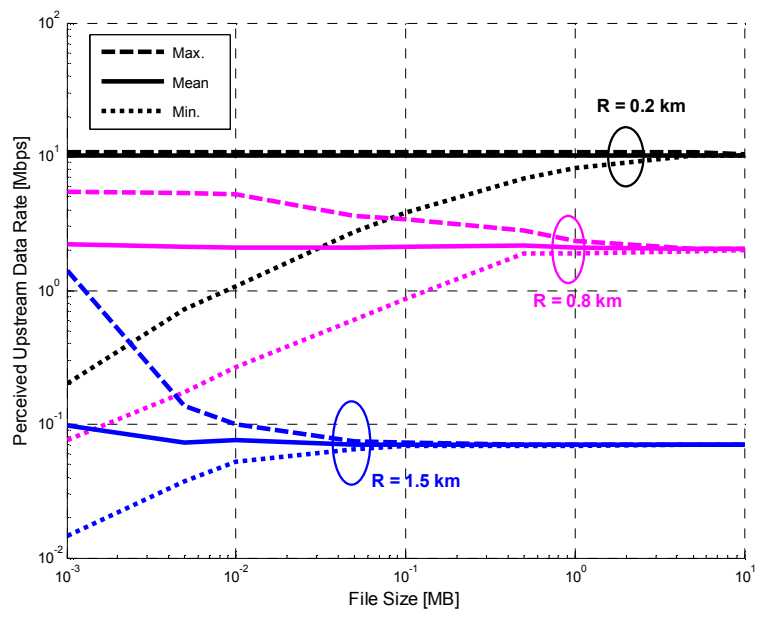

(a) Upstream

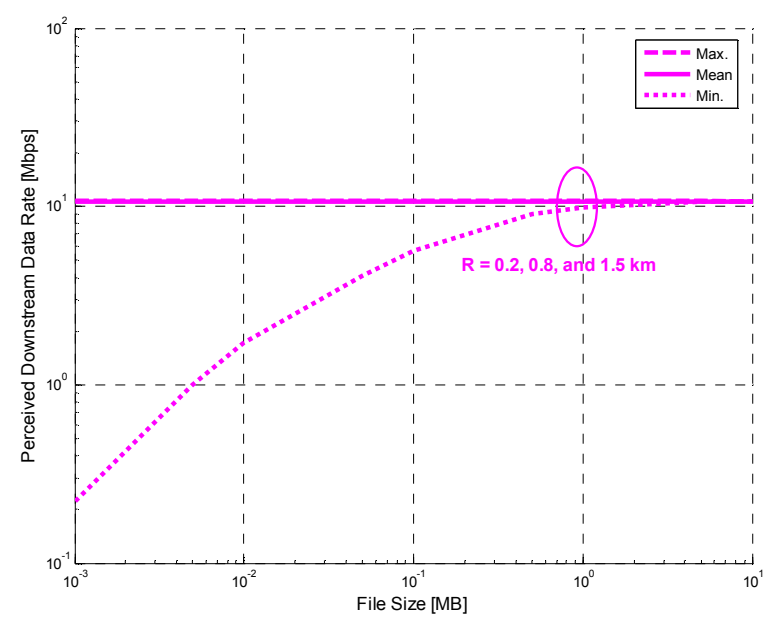

(b) Downstream

Figure 9 - Sensitivity of Perceived Secondary Data Rate on Secondary Cell Radius.

The effect of the other parameters (including transmit power, INR, and rotating period) on the fluctuations in downstream perceived data rate are similar to the effect of cell radius as shown in Fig. 9(b), hence, the results on fluctuations of perceived data rate in the downstream are omitted. For the upstream, Fig. 10 shows the perceived data rate as a function of file size when (a) the radar transmit powers are $0.025,0.45$, and 1.4 MW, (b) INR is $-13,-10$, and $-7 \mathrm{~dB}$, and (c) the rotating periods are $4,4.7$, and $6 \mathrm{~s}$; the results are from a cell $70 \mathrm{~km}$ away from the radar. Fig. 10 shows that changes in these three parameters have only marginal impact on the fluctuations in data rate perceived when files larger than $1 \mathrm{MB}$ are uploaded. Hence, when one adjusts these parameters within this reasonable range, a user transferring a large file would still experience insignificant fluctuations in perceived data rate. Note from Fig. 10(b) that, as we can expect from the sensitivity analysis on average extent of secondary transmissions, when a small number of users are assumed in a cell, changing radar INR would have no impact on the fluctuations in upstream perceived data rate.

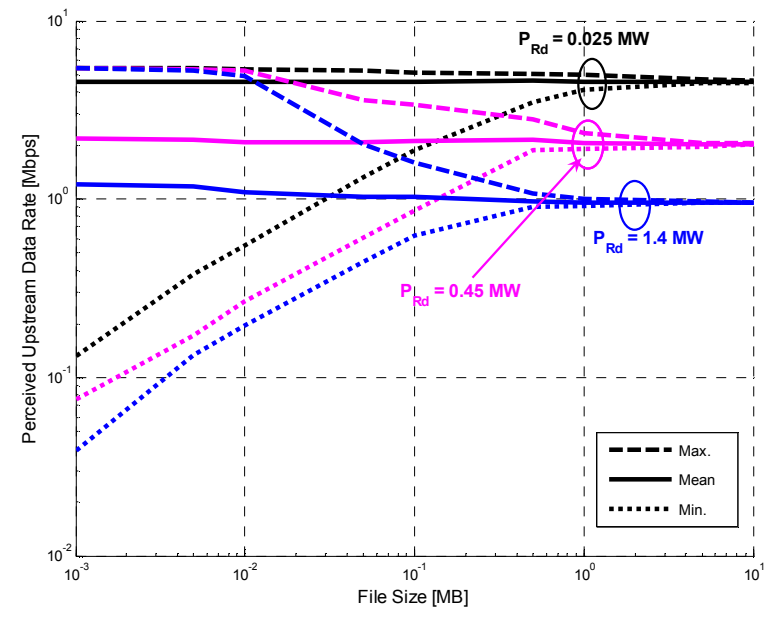

(a) on Radar Transmit Power 


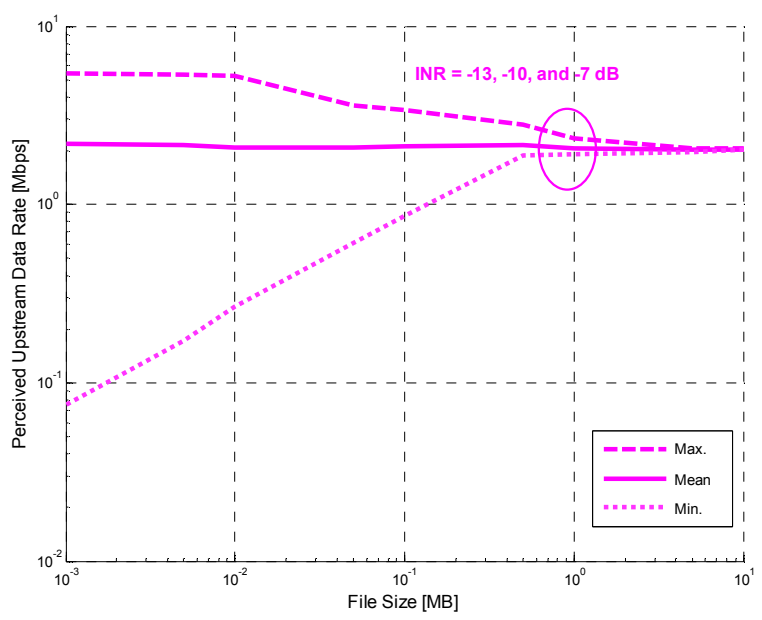

(b) on Radar Tolerable Interference to Noise Ratio (INR)

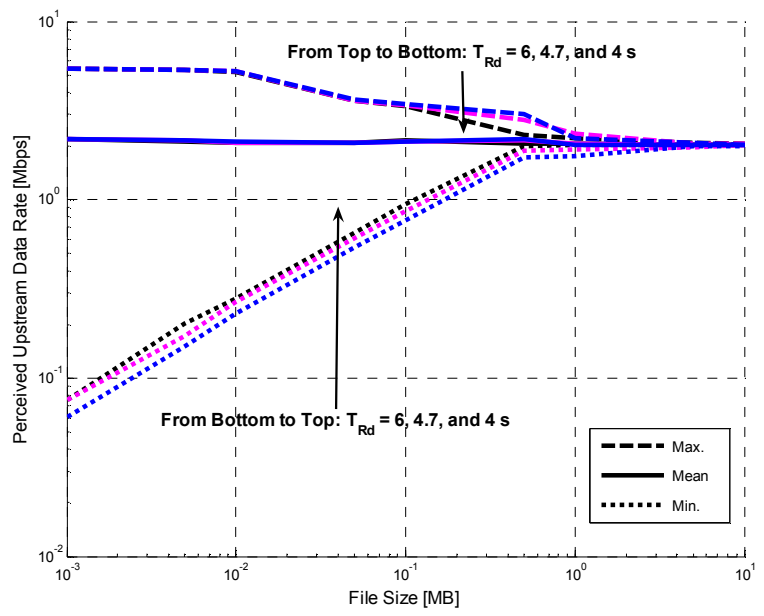

(c) on Radar Rotating Period

Figure 10 - Sensitivity of Perceived Secondary Data Rate in the Upstream.

\section{CONCLUSIONS}

We study opportunistic primary-secondary spectrum sharing with a rotating radar. A secondary device is allowed to transmit as long as the resulting interference does not exceed the tolerable level of the radar. The secondary system considered provides communications in non-contiguous cells around the radar.

This paper considers sharing between a radar and a cell, as might be appropriate if the secondary system provides broadband hotspots, or is a cellular system utilizing the shared spectrum when traffic temporarily exceeds the capacity of its dedicated spectrum. The model will be extended to multiple cells and radars in future work. Unlike existing models of sharing with radar, the proposed model allows secondary devices to adjust to variations in radar antenna gain as the radar's main beam rotates, thereby making extensive secondary transmissions possible, although with some interruptions and fluctuations. Thus, sharing spectrum with rotating radar is a promising option to alleviate spectrum scarcity. Additional technical and governance mechanisms are needed, e.g. to address interference from malfunctioning devices [6], [16].

It is also found that the secondary system will utilize spectrum more efficiently in the downstream than in the upstream, where efficiency may be measured in data rate per $\mathrm{MHz}$ of spectrum as compared to what can be achieved in dedicated spectrum, and fraction of time that a secondary device can transmit. Hence, spectrum sharing with radar would be more appropriate for applications that require more capacity in the downstream, which is a typical characteristic of many applications. However, if needed, the upstream rate could be increased by dedicating more spectrum to the upstream.

Moreover, with the dynamic sharing model considered, the secondary system can achieve extensive transmissions in large areas that otherwise would be unavailable with current approaches to sharing with radar which is more static. For example, with the base-case assumptions, a secondary device that does not adjust its transmit power as the radar's main beam rotates must be at least $286 \mathrm{~km}$ from the radar to prevent harmful interference. With the sharing model, at $27 \%$ of the distance at which secondary transmissions will not affect a radar, the secondary system can transmit all the time in the downstream with an average data rate almost equal to that achieved in dedicated spectrum, and roughly $90 \%$ of the time in the upstream with an average rate equal to $63 \%$ of the dedicated-spectrum data rate. It is also find that the secondary system can still achieve extensive transmissions even when key system parameters, including cell radius, radar transmit power, radar tolerable interference level and radar rotating period, change from the base-case assumptions.

Although average data rate is roughly the same for all file sizes, because of interruptions as the radar rotates, perceived data rate fluctuates for smaller files while appearing fairly constant for larger files. The magnitude of this fluctuation is also more noticeable when a secondary device far from the radar transmits small files. The fluctuations in perceived data rate make sharing spectrum with radar attractive for applications that can tolerate interruptions in transmissions, such as video on demand, peer-to-peer file sharing, and automatic meter reading, or applications that transfer large enough files so the fluctuations are not noticeable, such as song transfers. Moreover, even with changes in cell radius, radar transmit power, radar tolerable interference level, and radar rotating period, spectrum shared with radar is still attractive to these applications.

Especially for video on demand, because currently the application is the fastest rising traffic class in the Internet [29], it appears to be a very promising application that can share spectrum with radar. We have also found that this form of sharing works well with an application such as web browsing for which file transfer time rather than perceived data rate is the most appropriate performance measure. In contrast, spectrum shared with radar would be unattractive for interactive exchanges of small pieces of data, e.g., packets or files, of which instantaneous data rate matters, such as VoIP. 


\section{ACKNOWLEDGMENT}

The authors would like to thank Prof. Antonio A. Moreira, of Instituto Superior Técnico, for very helpful discussions on characteristics of radar and its antenna, and anonymous reviewers for their insightful comments that help improving the quality of this paper.

\section{REFERENCES}

[1] J.M. Peha, "Sharing spectrum through spectrum policy reform and cognitive radio," Proc. of the IEEE, vol.97, no.4, Apr. 2009, pp.708-19, (www.ece.cmu.edu/ peha/wireless.html).

[2] US National Telecommunications and Information Administration (NTIA), Presentation: spectrum with significant federal commitments, $225 \mathrm{MHz}-3.7 \mathrm{GHz}, 2009$.

[3] US NTIA, Federal Radar Spectrum Requirements, Special Publication 00-40, May 2000.

[4] R.P. Margie, "Efficiency, predictability, and the need for an improved interference standard at the FCC," Proc. of 31st Telecommun. Policy Research Conf. (TPRC), Arlington, VA, USA, Sep. 2003.

[5] US Federal Communications Commission (FCC), In the matter of Revision of Parts 2 and 15 of the Commission's Rules to Permit Unlicensed National Information Infrastructure (U-NII) devices in the 5 GHz band, $M O \& O$, ET Docket No. 03-122, June 2006.

[6] J.M. Peha, "Spectrum sharing in the gray space," to appear in Telecommunication Policy Journal.

[7] R. Saruthirathanaworakun, and J.M. Peha, "Dynamic primary-secondary spectrum sharing with cellular systems," Proc. of CrownCom, Cannes, France, June 2010, (www.ece.cmu.edu/ peha/wireless.html).

[8] M.J. Marcus, "Sharing government spectrum with private users: opportunities and challenges," IEEE Wireless Communications Magazine, vol.16, no.3, June 2009, pp. 4-5.

[9] L. Wang, J. McGeehan, C. Williams, and A. Doufexi, "Radar spectrum opportunities for cognitive communications transmission," Proc. of CrownCom, Singapore, May 2008.

[10] M.I. Rahman, and J.S. Karlsson, "Feasibility evaluations for secondary LTE usage in 2.7-2.9 GHz radar bands," Proc. of IEEE PIMRC, Toronto, Canada, Sep. 2011.

[11] 3GPP, Technical Specification 22.105: Services and service capabilities, version 9.1.0, Release 9, Oct 2010

[12] M.I. Skolnik, Introduction to Radar Systems, 3rd International Ed., McGraw-Hill, Singapore, 2001.

[13] ITU-R, Rec. M.1464-1: Characteristics of radiolocation radars, and characteristics and protection criteria for sharing studies for aeronautical radionavigation and meteorological radars in the radiodetermination service operating in the frequency band 2 700-2 900 $\mathrm{MHz}, 2003$.

[14] B. Bedford and F. Sanders, "Spectrum sharing and potential interference to radars," Proc. of ISART, Boulder, CO, USA, Feb. 2007.

[15] UK Office of Communications (Ofcom), Coexistence of $S$ Band radar systems and adjacent future services, Information Update, Dec. 2009.

[16] J.M. Peha, "Cellular systems and rotating radar using the same spectrum," Proc. of ISART, Boulder, CO, USA, July 2011, (www.ece.cmu.edu/ peha/wireless.html).

[17] N.M. Jacinto, Performance gains evaluation from UMTS/HSPA+ to LTE at the radio network level, M.Sc. Thesis, IST-Tech. Univ. Lisbon, Portugal, 2009.

[18] R.L. Hinkle, R. M. Pratt, and R. J. Matheson, Spectrum Resource Assessment in the 2.7 to $2.9 \mathrm{GHz}$ Band Phase II: Measurements and Model Validation (Appendix A-Antenna Characteristic Modeling), OT Report 76-97, Office of Telecommunications, US, Aug. 1976.

[19] H. Holma, P. Kinnunen, I.Z. Kovács, K. Pajukoski, K. Pedersen, and J. Reunanen, "Performance," in H. Holma and A. Toskala (ed.), LTE for UMTS: OFDMA and SC-FDMA Based Radio Access, John Wiley \& Sons, UK, 2009.

[20] The Netflix Blog: (http://blog.netflix.com/2008/11/encoding-forstreaming.html?CREATIVE $=\mathrm{n} \& \mathrm{KID}=\mathrm{k} 175726 \&$ USERID $=618506686$ \&SESSIONID=id_618506687, June 2011).

[21] A. Zambelli, IIS Smooth Streaming Technical Overview, White Paper, Microsoft Cooperation, USA, 2009.

[22] ITU-R, Rec. P.1546-4: Method for point-to-area predictions for terrestrials services in the frequency range $30 \mathrm{MHz}$ to $3000 \mathrm{MHz}, 2009$.
[23] T. Kurner, "Propagation Model for Macro-Cells," in E. Damosso, and L.M. Correia (ed.), COST 231 Final Report, European Communities, Brussels, Belgium, 1999

[24] R. Hallahan, and J.M. Peha, "Quatifying the cost of a nationawide public safety wireless network," Telecommunications Policy, vol.34, no.4, May 2010, pp. 200-20, (www.ece.cmu.edu/ peha/safety.html).

[25] ITU-R, Rec. P.1057-2: Probability distributions relevant to radiowave propagation modeling, 2007.

[26] J.T. Pinto, Assessment of multilateration telecommunication systems installed in NAV, M.Sc. Thesis, IST-Tech. Univ. Lisbon, Portugal, 2011.

[27] J.D. Parsons, The Mobile Radio propagation Channel, 2nd Ed., John Wiley \& Sons, Chichester, UK, 2000.

[28] S. Ramachandran, Web Metrics: Size and number of resources, Google, (http://code.google.com/speed/articles/web-metrics.html, June 2011).

[29] Cisco, Visual Networking Index: Global Mobile Data Traffic Forecast Update, 2010-2015, White Paper, Cisco, USA, Feb. 2011.

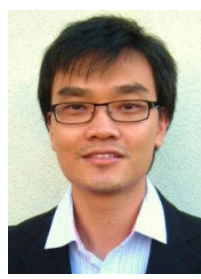

Rathapon Saruthirathanaworakun (S'11) received the B.Eng. and M.Eng degrees, both in electrical engineering, from Chulalongkorn University, Bangkok, Thailand.

Before pursuing Ph.D., he worked as an engineer for TOT public limited company, formerly Telephone Organization of Thailand, and was responsible for spectrum management and wireless engineering. Now, he is a Ph.D. candidate in Engineering and Public Policy, in dual Ph.D. program between Carnegie Mellon University, Pittsburgh, PA, USA, and Instituto Superior Técnico, Technical University of Lisbon, Portugal. His research topic is currently on spectrum management, spectrum sharing model, and theirs implications on related regulation and policy.

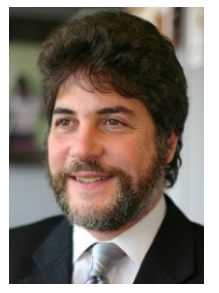

Jon M. Peha (S', M', SM', F'11) received the B.Sc. degree from Brown University, Providence, RI, and the $\mathrm{Ph} . \mathrm{D}$. degree in electrical engineering from Stanford University, Stanford, CA.

$\mathrm{He}$ is a Full Professor at Carnegie Mellon University. He served in the US Government in 2008-2011, first as Chief Technologist of the Federal Communications Commission, and then Assistant Director of the White House Office of Science \& Technology Policy where he focused on Communications and Research. At Carnegie Mellon, he is a Professor in the Dept. of Engineering \& Public Policy and the Dept. of Electrical \& Computer Engineering, and former Associate Director of the university's Center for Wireless \& Broadband Networking. He has served as Chief Technical Officer for three high-tech companies, and a member of technical staff at SRI International, AT\&T Bell Laboratories, and Microsoft. He has addressed telecom and e-commerce policy on legislative staff in the House Energy \& Commerce Committee and the Senate, and helped launch and lead a US Government interagency program to assist developing countries with information infrastructure. His research spans technical and policy issues of information networks, including spectrum management, broadband Internet, wireless networks, video and voice over IP, communications for emergency responders, universal service, secure Internet payment systems, online dissemination of copyrighted material, and network security. He is an IEEE Fellow, an AAAS Fellow, and a winner of the FCC's "Excellence in Engineering Award" and the IEEE TCCN Publication Award.

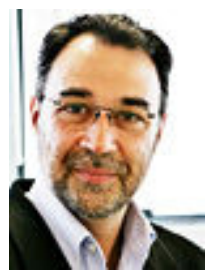

Luis M. Correia (S'85, M'91, SM'03) was born in Portugal, on 1958. He received the Ph.D. in Electrical and Computer Engineering from IST-TUL (Technical University of Lisbon) in 1991, where he is currently a Professor in Telecommunications, with his work focused in Wireless/Mobile Communications in the areas of propagation, channel characterisation, radio networks, traffic, and applications. He has acted as a consultant for Portuguese mobile communications operators and the telecommunications regulator, besides other public and private entities. Besides being responsible for research projects at the national level, he has been active in various ones within the European frameworks of RACE, ACTS, IST, ICT and COST (where he also served as evaluator and auditor), having coordinated two COST projects, and taken leadership responsibilities at various levels in many others. He has supervised more than $150 \mathrm{M}$.Sc. and $\mathrm{Ph} . \mathrm{D}$. students, having authored more than 300 papers in international and national journals and conferences, for which he has served also as a reviewer, 
editor, and board member, and edited 6 books. He was part of the COST Domain Committee on ICT. He was the Chairman of the Technical Programme Committee of several conferences, namely PIMRC'2002. He is part of the Expert Advisory Group and of the Steering Board of the European Net!Works platform, and was the Chairman of its Working Group on Applications. 